\title{
Liquid biopsy: Where did it come from, what is it, and where is it going?
}

If it seems like the term "liquid biopsy" is becoming as commonplace as "precision medicine," that's because it probably is. While the phrase has been around for many years, recent interest has dramatically increased in parallel with major scientific advances in the field. The term is generally thought of as it relates to measuring tumor cells or nucleic acids circulating in the blood. However, as the expression has grown in popularity, it has been applied to urine and saliva in addition to sets of protein markers, such as in the 4 Kscore which measures prostate specific antigen and related isoforms.

Defining what constitutes a liquid biopsy is important here. The term biopsy implies direct measurement of a tumor, so the liquid biopsy moniker should be restricted to tests with specificity approaching that of a tissue biopsy. $P C A 3$ is a long non-coding RNA measurable in urine and with strong performance characteristics for predicting the presence of prostate cancer. But with a specificity of approximately $75 \%$ at a commonly used cutoff of 35 , it is nowhere near a surrogate for an actual biopsy. Further, a test that is based solely on measuring elevated serum levels of a protein (or proteins) secreted by a tumor, may be a great circulating tumor marker but not represent a liquid biopsy. Alpha-fetoprotein and human chorionic gonadotropin, for example, do not constitute a liquid biopsy for testis cancer any more than the prostate health index (PHI) does for prostate cancer.

Notably, over the past several years, a number of exciting-and highly tumor-specific-approaches to performing liquid biopsies have been described. These methods are largely centered around the capture and analysis of circulating tumor cells (CTCs) and circulating tumor DNA (ctDNA) in the blood of patients with cancer. It is worth considering each of these approaches separately, as they provide different, and potentially complementary, information.
CTC technologies are not new, but there remains only one US Food and Drug Administration (FDA)-approved platform (CellSearch; Menarini-Silicon Biosystems, Bryn Athyn, PA, USA). Like CellSearch, most CTC-enrichment methodologies rely on epithelial cell capture. There is an abundance of data in breast, colon, and prostate cancer demonstrating that the presence of these circulating epithelial cells is associated with more aggressive disease [1]. However, many CTCs may have downregulated expression of epithelial cell surface markers used for capture (such as through the process of epithelial-mesenchymal transition), highlighting a well-known potential weakness of epithelial capture-based approaches. Even most of the recent and highly innovative microfluidic-driven approaches to CTC capture include some reliance on epithelial marker expression in defining CTCs. While such CTC selection strategies may be effective in the vast majority of cases, a more agnostic, morphologydriven approach may allow detection of a wider variety of CTCs. For example, recent work using the Epic Sciences CTC platform demonstrated that prostate cancer CTCs can possess a great deal of phenotypic heterogeneity [2]. This heterogeneity itself can serve as a prognostic marker, with metastatic castration-resistant prostate cancer (mCRPC) patients demonstrating decreased overall survival in the context of greater heterogeneity.

The tremendous challenges inherent to collecting clinically meaningful information from CTCs can't be overstated. A patient sample might have 10 CTCs in a background of $10^{6}$ white blood cells and $10^{9}$ red blood cells. With successful enrichment and isolation, however, there are numerous potential advantages of a CTC-based liquid biopsy. These advantages may be both logistical, such as the ease of collection and serial testing, and biological, as these CTCs may better reflect the whole-body burden of disease and provide a more encompassing snapshot than an isolated biopsy. Additionally, preservation of cellular contents by 
the cell membrane means gene expression information can be garnered from CTCs - even at the single cell level-in contrast to ctDNA-based liquid biopsy approaches. This is best exemplified by the work of Antonarakis and colleagues who demonstrated that expression of the AR-V7 splice variant in CTCs strongly predicts resistance to abiraterone and enzalutamide in mCRPC.

Given the significant technical and biological challenges of CTC characterization, there has also been great enthusiasm for ctDNA detection and analysis. The ctDNA itself comes from apoptosis and necrosis of tumor cells, as well as from tumor cell exosome excretion, and it can account for anywhere from $0 \%$ to $>50 \%$ of all detectable cell free DNA in circulation. In theory, ctDNA can be used to recapitulate existing precision medicine workflows, with analyses ranging from identification of point mutations to higher level assessment of copy number alterations and structural rearrangements. These assays often utilize polymerase chain reaction (PCR)-based approacheseffective for identifying tumor variants with known recurring mutations. Alternatively, next generation sequencing (NGS)-based techniques can be used to assay more genes and more mutations within each gene, along with copy number changes and gene fusions. NGS approaches are difficult, however, given the variable ctDNA content in the blood and potential need for expensive, ultra-deep sequencing to ensure detection of key alterations. We have recently proposed a stepwise approach, starting with ultra-low pass whole genome sequencing, as a potentially cost-effective strategy [3].

Critically, ctDNA does appear to be an accurate representation of the tumor itself, which is an integral requirement for any liquid biopsy. Wyatt et al. [4] performed targeted sequencing of ctDNA and matched metastatic tissue biopsies in 45 patients with mCRPC. Copy number profiles and other gene alterations were identified from ctDNA in the majority of patients and were highly concordant with tissue sequencing. Of particular interest, a number of patients harbored clinically relevant alterations in their ctDNA that were not identified in their tumor biopsy, potentially indicative of tumor heterogeneity that isn't captured by a needle core. There is already one FDAapproved ctDNA assay, the Cobas epidermal growth factor receptor (EGFR) Mutation Test (Roche, Basel, Switzerland). This PCR-based assay detects specific EGFR mutations and may be used to direct use of EGFR tyrosine kinase inhibitor therapy in non-small cell lung cancer. Notably, though, patients with a negative test are still required to undergo tissue biopsy due to concern of false negative results. In prostate cancer, there is now evidence that ctDNA can be used to identify alterations in BRCA2, ATM, TP53, and AR that are associated with de novo resistance to abiraterone and enzalutamide [5].

While these advances are encouraging, there is also reason for caution. Torga and Pienta [6] published a brief report after sending 40 paired patient samples for analysis on two CLIA-certified commercially available ctDNA platforms. The Guardant360 (Guardant Health, Inc., Redwood City, CA, USA) panel assess 73 genes while the PlasmaSelect (Personal Genome Diagnostics, Inc., Baltimore, MD, USA) test is a 64-gene panel. Findings for the 42 genes covered by both panels were compared, and, strikingly, there was little concordance between the two assays for the same patients. In this context, a potentially paradigm changing paper just published in Science deserves a similar level of caution. Cohen et al. [7] report on the CancerSEEK assay, which assesses for both ctDNA mutations and a panel of eight circulating protein markers. Developed as an early detection test for multiple cancer types (ovary, liver, stomach, pancreas, esophagus, colorectal, lung, and breast), the assay demonstrated a median sensitivity of $70 \%$ and a specificity $>99 \%$. While these data are exciting, the assay requires rigorous prospective study before being considered for clinical use.

Going forward, technical advances and clinical implementation of liquid biopsy approaches will likely mirror that of tissue-based platforms for precision medicine. As tumor gene alterations are identified that can guide therapy choice, CTC and ctDNA-based tests will be developed to assess for these changes in a simple blood draw. As these advances continue, however, the core EGAPP (Evaluation of Genomic Applications in Practice and Prevention) framework of establishing both validity and utility will be absolutely essential prior to wide adoption of these tests in the clinic. The blood profiling atlas in cancer (BloodPAC) is large public-private collaborative seeking to systematically address these issues in order to help safely bring liquid biopsies into routine clinical care. To quote my colleague and former American Society of Clinical Oncology president Daniel Hayes, MD, "a bad biomarker test is as bad as a bad drug." We need rigorous, prospective, randomized testing of these assays before we begin to use them to guide our clinical care.

\section{CONFLICTS OF INTEREST}

The author has nothing to disclose. 
Todd M. Morgan

ORCID: https:/orcid.org/0000-0002-1140-0603

Department of Urology, University of Michigan,

Ann Arbor, MI, USA

E-mail: tomorgan@med.umich.edu

\section{REFERENCES}

1. Singhal U, Wang Y, Henderson J, Niknafs YS, Qiao Y, Gursky A, et al. Multigene profiling of CTCs in mCRPC identifies a clinically relevant prognostic signature. Mol Cancer Res 2018;16:643-54.

2. Scher HI, Graf RP, Schreiber NA, McLaughlin B, Jendrisak A, Wang Y, et al. Phenotypic heterogeneity of circulating tumor cells informs clinical decisions between AR signaling inhibitors and taxanes in metastatic prostate cancer. Cancer Res 2017;77:5687-98.
3. Hovelson DH, Liu CJ, Wang Y, Kang Q, Henderson J, Gursky A, et al. Rapid, ultra low coverage copy number profiling of cellfree DNA as a precision oncology screening strategy. Oncotarget 2017;8:89848-66.

4. Wyatt AW, Annala M, Aggarwal R, Beja K, Feng F, Youngren J, et al. Concordance of circulating tumor DNA and matched metastatic tissue biopsy in prostate cancer. J Natl Cancer Inst 2017;109.

5. Annala M, Vandekerkhove G, Khalaf D, Taavitsainen S, Beja K, Warner EW, et al. Circulating Tumor DNA genomics correlate with resistance to abiraterone and enzalutamide in prostate cancer. Cancer Discov 2018;8:444-57.

6. Torga G, Pienta KJ. Patient-paired sample congruence between 2 commercial liquid biopsy tests. JAMA Oncol 2018;4:868-70.

7. Cohen JD, Li L, Wang Y, Thoburn C, Afsari B, Danilova L, et al. Detection and localization of surgically resectable cancers with a multi-analyte blood test. Science 2018;359:926-30. 